\title{
EDUCATIONAND TRAINING An audible patient voice: How can we ensure that patients are treated as partners in their own
} safety?

\author{
Authors: Christian P Subbe, ${ }^{A}$ Saleyha Ahsan, ${ }^{B}$ Lowri Smith ${ }^{C}$ and Johanna Frey Renggli ${ }^{D}$
}

\begin{abstract}
How can patients and their relatives make their concerns heard by healthcare professionals? Many serious adverse events are preceded by patients' worry and concern. This article explores changes in the structures and processes of healthcare that might facilitate safer systems. One important tool might be the ability of patients to become equal partners in the recording of their clinical history.
\end{abstract}

KEYWORDS: patient centred, patient safety, adverse event

DOI: 10.7861/fhj.2021-0131

\section{The experience of not being heard}

Dr Saleyha Ahsan is a doctor working in emergency medicine. She experienced the powerlessness of being a relative when her mother was admitted to hospital. Despite being a doctor (and an articulate renowned broadcast journalist), her concerns about the dramatic deterioration of her mother went unheard.

Alison Phillips suffered serious injuries as part of a road traffic accident. On several occasions during her hospital stay, she and those close to her identified serious problems (that resulted in prolonged intensive care stays) well before her clinical team had. ${ }^{2}$

Lowri Smith has a complex congenital heart condition and has been under close supervision by a tertiary centre for her entire life. In 2018, she identified an infected central line but had difficulties to gain support with getting it removed (Box 1).

What is common to all these experiences (and many others) is that patients and those close to them had prior experience and were applying their learning but had great difficulties to be heard as equitable partners in clinical safety during episodes of serious illness, and this despite being able to articulate this knowledge. Clinicians dismissed their evidence and expertise and were able to do so effortlessly.

Authors: ${ }^{\text {A }}$ senior clinical lecturer, School of Medical Sciences, Bangor, UK; ${ }^{\mathrm{B}}$ emergency physician and PhD student, Cambridge University, Cambridge, UK; ${ }^{\text {C }}$ patient representative, Ruthin, UK; ${ }^{\text {Demergency }}$ physician, University Hospital of Basel, Basel, Switzerland

\section{Quality improvement beyond co-production}

The reasons behind the observations earlier are complex and are sometimes described with the term 'culture'. Culture manifests itself in beliefs and behaviour: beliefs about what is right, acceptable, tolerable and that are difficult to observe and quantify; and behaviours that can be observed and quantified. ${ }^{3}$ The usual response to 'cultural' challenges, complaints or adverse events are educational interventions. ${ }^{4}$ It would seem that after decades of a focus on better communication, 'what matters to you', co-production and shared decision making, something more transformational might be required $?^{5-7}$ That would be something that shifts the balance of available information, intervention and governance (and power) towards those that are affected by medical error and adverse events.

Quality improvement can be described in terms of outcomes, processes that are required to deliver those and the structures that enable them. If patient safety is going to improve, it will require deeper understanding about the processes and structures that enable the current level of care and the changes that would deliver more reliably safe care.

\section{A narrowing gap between professionals and patients}

In many industries, customers have gained access to tools to that were previously the prerogative of experts, from reading of gas meters to building furniture. This has enabled a shift in the relationship between customers and experts. In medical care, cautious signs of such a shift are visible: using vital sign monitors during the COVID-19 pandemic, checklists for safer surgery, and accessing clinical records to document their own symptoms and views. ${ }^{8-10}$ A rising number of patients with chronic healthcare conditions in the UK are mastering the self-administration of complex and time sensitive medication at home while being wary that they are denied the ability to exercise this task in hospital. ${ }^{11}$ In all these instances, the gap between patients and healthcare professionals is narrowing.

\section{An audible and readable patient voice}

Taking a history is another pivotal step in the clinical process, for medical emergencies presenting to hospital, an extended history taking is often warranted. Against the backdrop of rising numbers of emergency visits, in recent years there has been 


\section{Box 1. Lowri Smith's experience}

In 2018, I was a patient in the intensive care unit (ICU). At the time, it was a precautionary measure following a lengthy and complex cardiac procedure via the jugular vein. I was an ICU patient for 5 days and intubated for 1-2 days. I had a central line place in my neck delivering antibiotics and other important medication. I have a horrific history of infection having had sepsis, which travelled to my heart resulting in endocarditis. The endocarditis destroyed my mitral valve; as a result, I'm always cautious of possible infection.

After 3-4 days in the ICU, I noticed that the central line felt warm, a sure sign of an infection brewing. I was immediately suspicious and mentioned it to my nurse. She stated that, as it was a 'bank holiday', the best she could do was swab the site, send it to the lab and wait 2-3 days for a result. I wasn't happy with her response but let her take a swab. What she didn't know is that I never give up that easily even when I'm lying in a hospital bed. Waiting 2-3 days means any infection would be circulating in my bloodstream by then.

There was another nurse, who I had observed for some time, caring for the patient opposite me. She appeared to be very experienced. Her patient was receiving extra-corporal membrane oxygenation, and the nurse was constantly talking with her patient. I managed to get her attention; I explained my concerns over the central line, possible infection and my very real history of septic shock and endocarditis. After examining the site, she agreed with my observation and alerted a nearby anaesthetist. The central line was immediately removed, and the anaesthetist inserted a canula into my hand because I still needed intravenous medications. Had they waited 2-3 days, they could potentially have been dealing with something far more serious. We all know that prevention is always better than cure. I don't know why my own nurse was reluctant to take the necessary action.

Regardless of your level of experience, please don't dismiss a patient's concern. Patients with long-term health conditions are often experts in their own conditions. They have picked up a lot of knowledge during their patient journey and their instincts are often spot on. They will be able to tell you what is normal for them and provide a good history. Their 'normal' won't necessarily reflect what's in the textbook, and that's okay. When a patient raises a concern, please take it seriously and, if needed, seek the opinion of their specialist team. Due to my medical history and cardiac condition, my risk of infection is high, and the potential consequences could, according to my specialist team, easily be fatal. The source of my sepsis (in 2003) was never established and could literally have been 'picked up' anywhere. When you are ill and in receipt of copious amounts of sedatives and other strong drugs, it takes an enormous amount of effort to do even the smallest thing, including stringing together a sentence. It is a time when even the most confident person is very vulnerable. You should not have to think about advocating for your own safety as a patient.

research efforts to use automated tools for history taking. The feasibility of such tools has been demonstrated and a team from University of Basel has shown the ability of a history taking app in gathering an extended patient history (including personal history, allergies, systematic history, risk factors etc). ${ }^{12,13}$ Another team in Toronto was able to identify ambulatory patients significantly quicker than the routine nurse-initiated patient identification using a self-check-in kiosk. ${ }^{14}$ Automated tools have also been shown to reduce the waiting time before seeing a healthcare professional in the emergency department. ${ }^{15} \mathrm{~A}$ group in Sweden is currently investigating the use of a self-reported history taking for patients with acute chest pain collecting data to assess chest pain according to the HEART score. ${ }^{16}$

Self-reported history taking tools could aid a shift in the relationships between healthcare professionals and patients and an increase in the time for direct patient contact, thoughtful communication and subsequently improved patient care. Implementation at scale in large systems and over long periods of time might be required to explore the real effects for patients and the safety of their care.

In this edition of the Future Healthcare Journal, readers will find examples on how more reliable safe care might be practically achieved by and with patients during major surgery, critical deterioration or even routine care. The impact of the described interventions has often not been evaluated at scale yet, but the philosophy and practice are aligned with a vision or modern healthcare where patients and those close to them are equitable partners with their clinicians, with the ability to change the course of their care in real time.

Whether these tools allow true listening to patients and those close to them, and change some of the culture and hierarchical thinking that has dominated medical care and hindered breakthroughs for patients' safety, remains to be seen.

\section{Acknowledgements}

Thanks to Alison Phillips for her testimony and contribution to the paper.

\section{References}

1 Dispatches. Covid critical: a doctor's story: Dispatches. All 4, 2021. www.channel4.com/programmes/covid-critical-a-doctors-storydispatches [Accessed 04 July 2021].

2 Health Service Journal Events. HS] Patient Safety Congress 2018 - Alison Phillips Story. YouTube, 2019. www.youtube.com/ watch?v=RPIBwk_V99o\&ab_channel=HealthServiceJournalEvents [Accessed 04 July 2021].

3 Wakefield JG, McLaws ML, Whitby M, Patton L. Patient safety culture: Factors that influence clinician involvement in patient safety behaviours. Qual Saf Health Care 2010;19:585-91.

4 Cohen J, Patel S. A medical department with memory: learning from adverse events so history doesn't repeat itself. BMJ Opinion 2021. https://blogs.bmj.com/bmj/2021/05/21/a-medical-departmentwith-memory-learning-from-adverse-events-so-history-doesntrepeat-itself [Accessed 11 July 2021].

5 Healthcare Improvement Scotland. What matters to you? Healthcare Improvement Scotland, 2016.

6 Voorberg WH, Bekkers VJJM, Tummers LG. A systematic review of co-creation and co-production: embarking on the social innovation journey. Public Manag Rev 2015;17:1333-57.

7 The Health Foundation. Implementing shared decision making. London: The Health Foundation, 2013.

8 Thornton J. The 'virtual wards' supporting patients with COVID-19 in the community. BMJ 2020;369:m2119.

9 Harris K, Søfteland E, Moi AL et al. Patients' and healthcare workers' recommendations for a surgical patient safety checklist A qualitative study. BMC Health Serv Res 2020;20:1-10. 
10 Wyatt J, Sathanandam S, Rastall P, Hoogewerf J, Wooldridge D. Personal health record (PHR) landscape review. Royal College of Physicians, 2016.

11 Stafford M, Steventon A, Thorlby R et al. Briefing: Understanding the health care needs of people with multiple health conditions. The Health Foundation, 2018.

12 Zakim D. Development and significance of automated historytaking software for clinical medicine, clinical research and basic medical science. J Intern Med 2016;280:287-99.

13 Renggli JF, Eken C, Siegrist V et al. Usability of a web-based software tool for history taking in the emergency department. Acute Med 2020;19:131-7.

14 Coyle N, Kennedy A, Schull MJ et al. The use of a self-check-in kiosk for early patient identification and queuing in the emergency department. CJEM 2019;21:789-92.
15 Mahmood A, Wyant DK, Kedia S et al. Self-check-in kiosks utilization and their association with wait times in emergency departments in the United States. J Emerg Med 2020;58:829-40.

16 Brandberg H, Sundberg CJ, Spaak ] et al. Use of self-reported computerized medical history taking for acute chest pain in the emergency department - the Clinical Expert Operating System Chest Pain Danderyd Study (CLEOS-CPDS): prospective cohort study. J Med Internet Res 2021;23:e25493.

Address for correspondence: Dr Christian P Subbe, School of Medical Sciences, Brigantia Building, Penrallt Road, Bangor, Gwynedd LL57 2AS, UK.

Email: c.subbe@bangor.ac.uk

Twitter: @csubbe 